\title{
T-shaped Peptide Amphiphiles Self Assemble into Nanofiber Networks
}

\author{
Funmilola A. Fisusi ${ }^{\mathrm{a}, \mathrm{d}}$, Rebecca Notman ${ }^{\mathrm{b}}$, Louis A. Granger ${ }^{\mathrm{b}}$, John P. Malkinson ${ }^{\mathrm{a}}$, Andreas G. \\ Schätzlein $^{\mathrm{a}, \mathrm{c}}$, and Ijeoma F. Uchegbu ${ }^{\mathrm{a}, \mathrm{c} *}$
}

${ }^{a}$ UCL School of Pharmacy, Brunswick Square London, WC1N 1AX, United Kingdom; ${ }^{b}$ Department of Chemistry and Centre for Scientific Computing, University of Warwick, Coventry, CV4 7AL, United Kingdom; ${ }^{c}$ Nanomerics Ltd, 1394 High Road, London N20 9YZ, United Kingdom; ${ }^{d}$ Drug Research and Production Unit, Faculty of Pharmacy, Obafemi Awolowo University, Ile-Ife, Nigeria.

\begin{abstract}
Background Conventional nanofiber forming peptide amphiphiles comprise a beta sheet forming, short peptide sequence with an alkyl chain attached at one terminus. We report the self-assembly of a peptide amphiphile possessing a mid-chain located alkyl substituent (a T-shaped peptide amphiphile) into nanofiber networks.

Method Peptide synthesis was carried out using standard 9-fluorenylmethoxycarbonyl solid phase peptide synthesis protocols, followed by covalent attachment of the alkyl chains to yield target peptide amphiphiles. Self-assembly was then studied using electron microscopy and coarse grained molecular dynamics simulations.

Results T-shaped peptide amphiphiles self-assembled into nanofibers just like linear peptide amphiphiles, but then unlike linear peptide amphiphiles, T-shaped peptide amphiphiles formed inter-fiber associations and ultimately nanofiber networks.

Conclusion Changing the position of the alkyl chain in a peptide amphiphile from the terminal end of the peptide to the middle part of the peptide, to form a T-shaped peptide amphiphile, does not disrupt the molecular interactions required for the self-assembly of the peptide amphiphiles into nanofibers.
\end{abstract}

Keywords: Peptide amphiphiles, self-assemblies, nanofibers, networks, beta sheet, peptide synthesis, molecular dynamics simulations, drug delivery.

\section{INTRODUCTION}

Peptide amphiphiles (PAs) are a class of materials that self-assemble into nanofibers, provided that certain architectural and physical chemistry conditions are met [1]. PAs generally comprise an alkyl chain attached at the end of a short peptide sequence, with the peptide sequence being capable of forming intermolecular hydrogen bonds, as intermolecular hydrogen bonding drives self-assembly into nanofibers through the formation of hydrophobic associations between the alkyl chains and beta $(\beta)$-sheets from the peptide sequences $[2,3]$. PA nanofibers in which the PAs possess alkyl substituents located at positions other than the termini of the molecule, such as those having a midchain located alkyl substituent (T-shaped PAs) have not been reported previously and this is the focus of the current report.

*Address correspondence to this author at the UCL School of Pharmacy, Brunswick Square, London, UK; Tel: +44 207753 5997; Fax: +44 207753 5942; E-mail: ijeoma.uchegbu@ucl.ac.uk.
The self-assembly of linear PAs into nanofibers, in aqueous media, as opposed to classical spherical aggregates, is governed by: a) the hydrophobic interaction of the alkyl tails and $b$ ) hydrogen bonding among the $\beta$-sheet forming peptide segments $[2,3]$. The formation of $\beta$-sheets drives the selfassembly away from spherical aggregates and towards cylindrical self-assemblies as hydrogen bonding between peptide sequences prevents the amphiphiles from adopting a cone shaped (suitable for spherical aggregates) structure. This is in keeping with Israelachvili's critical packing parameter theory [4].

Introducing charged amino acids reduces aggregation and/ or results in the formation of spherical or globular aggregates depending on the $\mathrm{pH}$ and amino acid sequence which in turn determines the charge distribution, with spherical aggregation due to the electrostatic repulsion between charged amino acid residues [5]. 
Consequently, the final structure and properties of the selfassembly (its size, shape/ interfacial curvature) is a reflection of the interplay between these attractive and repulsive forces $[6,7]$. It thus follows that PA self-assembly may be controlled by factors such as the amino acid sequence, concentration of the peptide, $\mathrm{pH}$ of the solution, temperature, ionic strength and nature of the solvent $[7,8]$.

PA nanofibers have found application in various disciplines [9] including tissue engineering [2, 10, 11] drug delivery [12] and transplantation [13, 14]. For tissue engineering cell scaffold applications, a nanofiber network would be beneficial.

\section{MATERIALS AND METHOD}

\section{Synthesis of PAs}

PAs were synthesized by 9-fluorenylmethoxycarbonyl (Fmoc) solid phase methods (Scheme 1). See Supplementary material for full details of the methods.

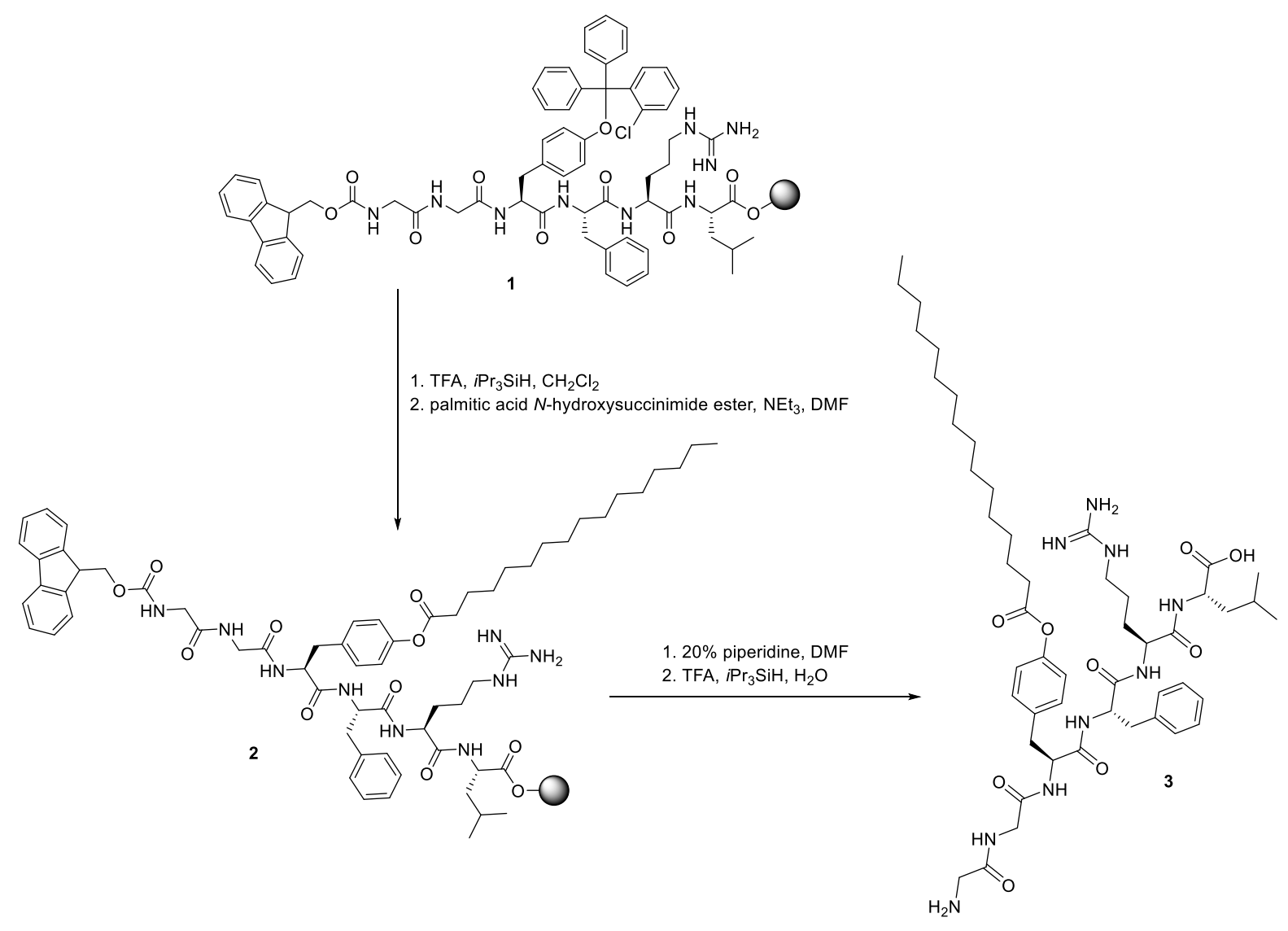

\section{Preparation of nanofibers}

Nanofibers were prepared by probe sonication of the peptides in aqueous media. Electron micrographs of PA dispersions were recorded using scanning and transmission electron microscopes.

Coarse grained molecular dynamics (CGMD) simulations were performed to model the self-assembly of the linear PA and T-shaped PA in water. To be able to assess the microsecond timescales required for observation of selfassembly, the MARTINI 2.1 coarse-grained (CG) potential for lipids [15] and proteins [16] were employed, with slight refinement which made it possible to capture the chemistry of the PAs more accurately. See Supplementary material for full details of the methods.

\section{RESULTS and DISCUSSIONS}

For the first time, we present data on the self-assembly into nanofibers of a PA possessing a mid-chain located alkyl substituent [GGY(O-Palm)FRL, 3; a T-shaped PA ("T"$\mathrm{PA})$ ], showing that the beta sheet formation is possible with these T-shaped peptides (Fig. 1a and 2a). Moreover, spectroscopic data (FTIR and fluorescence) obtained for the T-shaped PA showed that this peptide formed beta-sheets similar to the linear PA (see Supplementary material for full details). The T-shaped PA also formed inter-fiber associations and ultimately a nanofiber network at a concentration as low as $0.1 \% \mathrm{w} / \mathrm{v}$ (Fig. 1a and 2a). The control linear PA, possessing a terminal alkyl chain $(\mathrm{Y}(\mathrm{O}-$ Palm)GGLRF; "L"-PA), formed nanofibers but did not form nanofiber networks (Fig. $1 \mathrm{~b}$ and $2 \mathrm{~b}$ ). 


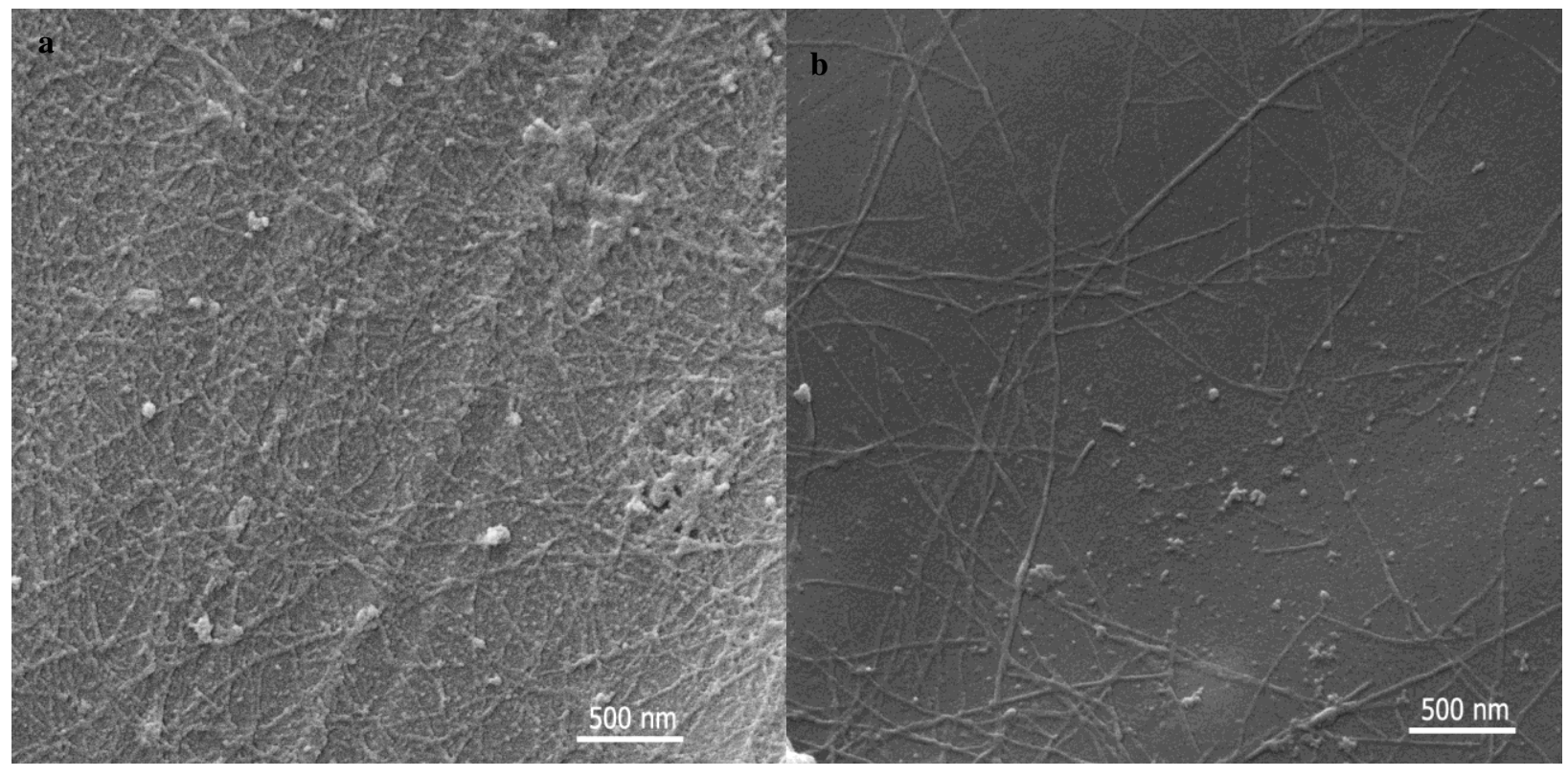

Fig. 1. Scanning electron micrograph of (a) T-shaped PA nanofiber networks, "T"-PA (0.1\% w/v); (b) Linear PA nanofibers, "L"-PA $(0.1 \% \mathrm{w} / \mathrm{v})$. PAs in double deionized water, vortexed for 30 seconds then probe sonicated (QSonica sonicator, Connecticut, USA) on ice for 15 minutes at amplitude of $15 \%$ of maximum output. The sample was diluted 10 times with double deionized water prior to imaging.

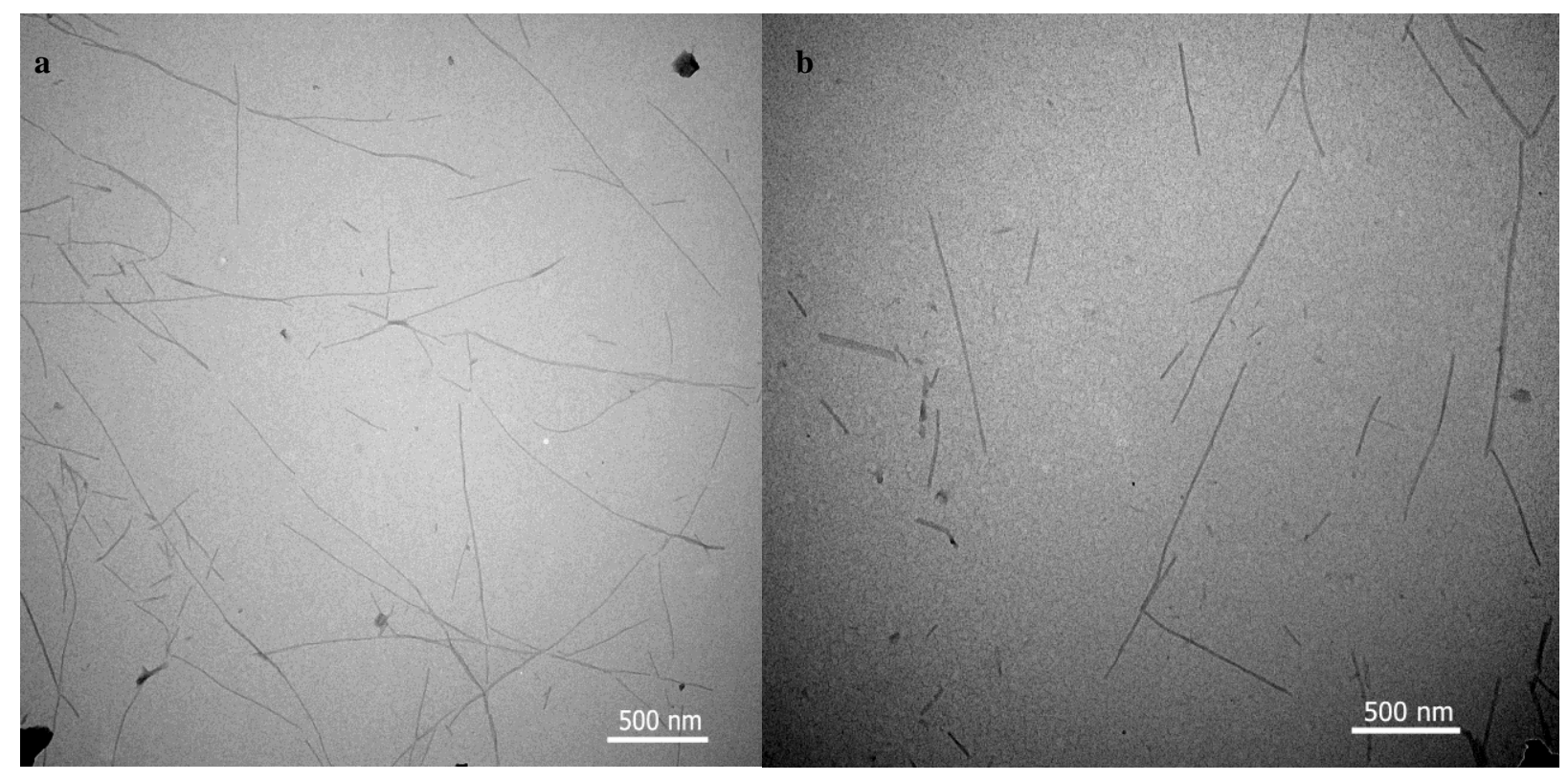

Fig. 2. Transmission electron micrograph of (a) T-shaped PA nanofiber networks, "T"-PA (0.1\% w/v); (b) Linear PA nanofibers, "L"-PA $(0.1 \% \mathrm{w} / \mathrm{v})$. PAs in double deionized water, vortexed for 30 seconds then probe sonicated (QSonica sonicator, Connecticut, USA) on ice for 15 minutes at amplitude of $15 \%$ of maximum output. 
Electron micrographs (Fig. 1 and 2) showed that both linear and T-shaped PAs self-assemble into nanofibers at a concentration of $0.1 \% \mathrm{w} / \mathrm{v}$ and the $\mathrm{T}$-shaped PAs formed dense nanofiber networks (Fig. 1a), while the linear PAs formed individual nanofibers with very few inter-fiber associations (Fig. 1b).
Furthermore, CGMD simulations of the self-assembly process for both PAs revealed that both PAs formed high axial ratio self-assemblies (Fig. 3) and that the T-shaped PAs self-assembled into branched nanofiber assemblies in all three simulation runs (see Fig. S12), whereas the linear PAs only showed some evidence of branching in one out of the three simulation runs (Fig. S14).

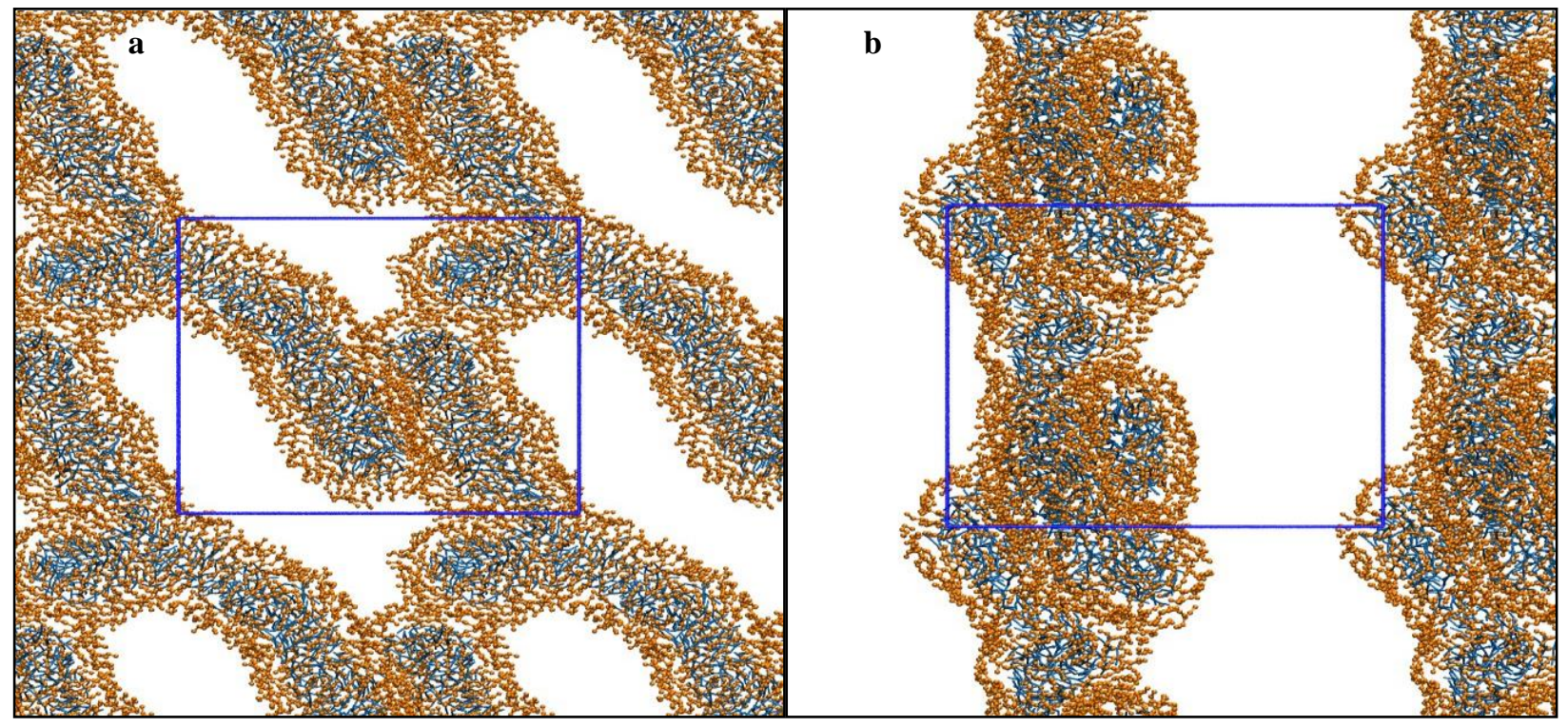

Fig. 3. (a) Snapshot from the simulation of nanofiber formed through self-assembly of "T"-PA (simulation time $>5 \mu \mathrm{s})$, (b) Snapshot from the simulation of nanofiber formed through self-assembly of "L"-PA ( backbone, blue = alkyl chain; amino acid side chains, water and ion molecules have been deleted from the image for clarity.

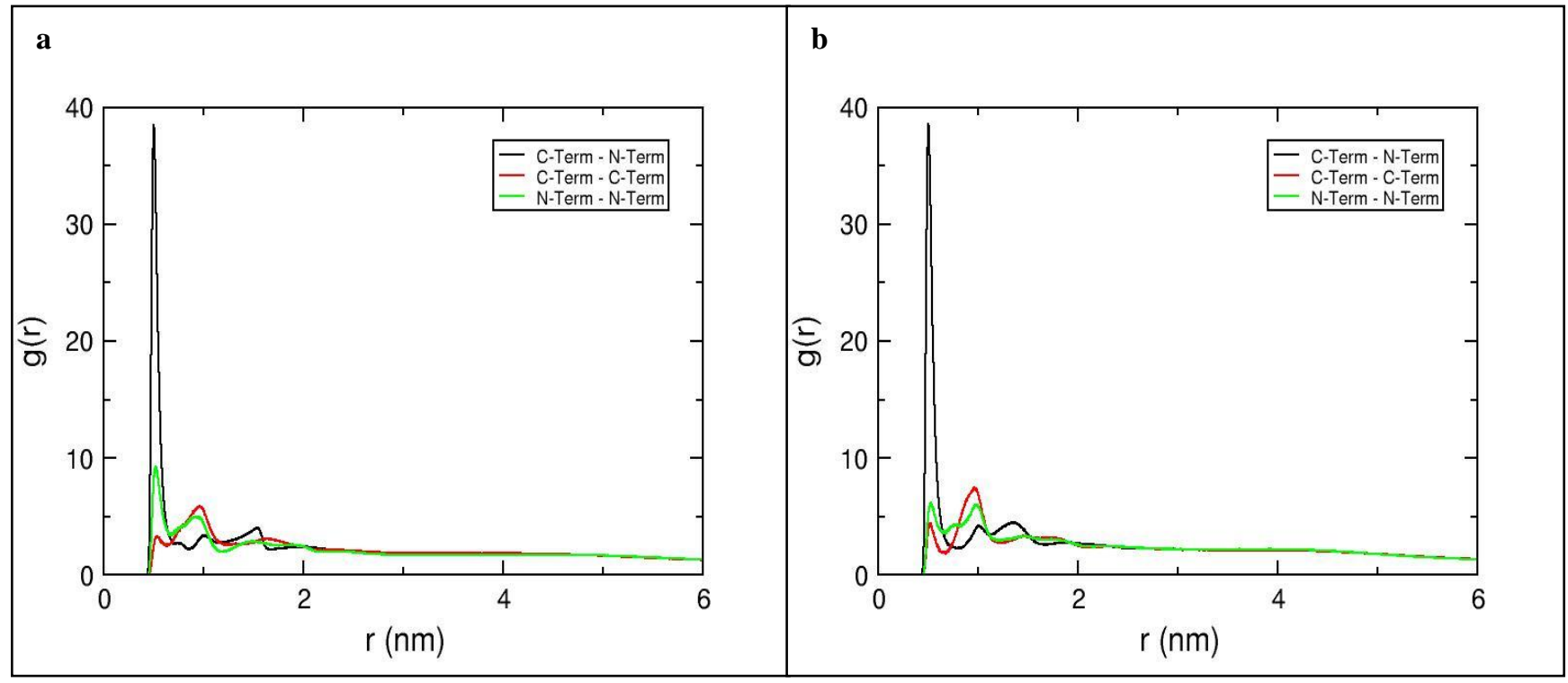

Fig. 4. (a) Radial distribution function for "T"-PA, (b) Radial distribution function for "L"-PA

Key: $r$ is the distance in $\mathrm{nm} ; \mathrm{g}(\mathrm{r})$ is the probability of finding an atom within the distance tested. 
In addition, the radial distribution functions (RDFs) for " $T$ "PA and "L"-PA suggest the formation of antiparallel $\beta$ sheets by both PAs (Fig. 4). The RDFs obtained for both PAs suggest the formation of antiparallel $\beta$-sheets as the peak obtained for the interaction of the $\mathrm{C}$ - terminus and the $\mathrm{N}$ - terminus is sharp, indicating that there is a strong interaction as would be expected for atoms in close proximity [17].

The molecular simulations data thus confirmed the formation of $\beta$-sheets by both the linear and T-shaped PAs and the tendency for T-shaped PAs to form nanofiber networks via inter-fiber associations.

This is the first report of the formation of nanofibers by Tshaped peptide amphiphiles and our data indicate that the peptide epitope is still able to engage in inter-peptide noncovalent interactions (presumably hydrogen bonding and beta sheet formation) even with the T-shaped architecture.

Relatively low concentrations $(0.25-0.5 \% \mathrm{w} / \mathrm{v})$ of ionic PAs had been reported to self-assemble into nanofiber networks as a result of the lowering of the $\mathrm{pH}$ or addition of metal ions $[14,18]$. Since network formation by PAs is induced by lowering the $\mathrm{pH}$ or the addition of metal ions, this implies that their self-assembly is initiated by the screening of their charges by counter ions in the medium, leading to a reduction in the repulsive forces. Subsequently, other non-covalent attractive forces such as van der Waals, hydrophobic interactions and hydrogen bonding promote close association of the nanofibers to form the nanofiber networks [18].

Therefore, nanofiber network formation is governed by the chemistry of each individual PA and the extent that this chemistry leads to inter-fiber associations. Chemistries that promote hydrophobic interactions including attraction between hydrophobic tails and hydrophobic amino acids and amino acid interactions including hydrogen bonding or van der Waals attractive forces will promote nanofiber network formation $[14,18]$. We add to this list of interactions - the bridging of two nanofibers by one PA molecule (due to the PA architecture) as is envisioned to be happening in this case (Fig. 3a), which then ultimately leads to the formation of branched nanofibers and nanofiber networks (see Fig. S12). Our study is the first to report the formation of nanofiber networks by T-shaped PAs, the formation of nanofiber networks in plain aqueous medium (neutral $\mathrm{pH}$ and without the addition of metal ions) and at an extremely low PA concentration $(0.1 \% \mathrm{w} / \mathrm{v}, 1.05 \mathrm{mM})$. This network formation is governed by the PA architecture.

The formation of nanofibers, which impede the thermal motion of the solution and resist external shear stress eventually leads to the transition from the liquid state to a self-supporting gel in such PAs [18].

\section{CONCLUSION}

We have demonstrated that changing the position of the alkyl chain in a PA from the terminal end of the peptide to the middle part of the peptide, to form a T-shaped PA, does not disrupt the molecular interactions required for the selfassembly of the PAs into nanofibers. Additionally, the Tshaped PA forms nanofiber networks, while the linear PA forms mostly single nanofibers, and this network formation is attributable to the molecular architecture of the T-shaped PA. Such nanofiber networks may find applications in drug delivery or tissue engineering.

\section{CONFLICT OF INTEREST}

The authors confirm that this article content has no conflict of interest.

\section{ACKNOWLEDGEMENTS}

Funmilola A Fisusi and John P. Malkinson performed peptide synthesis and purification; Rebecca Notman and Louis A. Granger performed CGMD simulations; Andreas G. Schätzlein and Ijeoma F. Uchegbu designed the study. All authors participated in writing the manuscript.

Funmilola A Fisusi received an Academic Staff Training and Development Grant from the Tertiary Education Trust Fund (TETFund), Nigeria for Ph.D. studies. Louis A. Granger received the University of Warwick Undergraduate Research Scholarship Scheme bursary to carry out the molecular simulations. Rebecca Notman is a recipient of the Royal Society University Research Fellowship. We acknowledge University College London for funding and the Centre for Scientific Computing, University of Warwick, for the provision of computing facilities.

\section{SUPPLEMENTARY MATERIAL}

Synthesis and characterization of the T-shaped PA and linear PA; beta sheet characterization of T-shaped PA and linear PA; mass spectra; proton $\left({ }^{1} \mathrm{H}\right)$ nuclear magnetic resonance (NMR) spectra; transmission and scanning electron micrographs; Fourier transform infrared (FTIR) and fluorescence spectroscopy data of peptide nanofibers (PDF). 


\section{REFERENCES}

[1] Ayres CE, Jha BS, Sell SA, Bowlin GL, Simpson DG. Nanotechnology in the design of soft tissue scaffolds: Innovations in structure and function. Wiley Interdiscip Rev Nanomed Nanobiotechnol 2010; 2(1): 20-34.

[2] Hartgerink JD, Beniash E, Stupp SI. Self-assembly and mineralization of peptide-amphiphile nanofibers. Science 2001; 294(5547): 1684-8.

[3] Mazza M, Notman R, Anwar J, et al. Nanofiber-based delivery of therapeutic peptides to the brain. ACS Nano 2013; 7(2): 1016-26.

[4] Israelachvili J. Intermolecular and surface forces. 3rd ed. Amsterdam, Academic Press; 2011.

[5] Jun S, Hong Y, Imamura H, Ha BY, Bechhoefer J, Chen P. Self-assembly of the ionic peptide eak16: The effect of charge distributions on self-assembly. Biophys J 2004; 87(2): 1249-59.

[6] Paramonov SE, Jun HW, Hartgerink JD. Self-assembly of peptide-amphiphile nanofibers: The roles of hydrogen bonding and amphiphilic packing. J Am Chem Soc 2006; 128(22): 7291-8.

[7] Cui H, Webber MJ, Stupp SI. Self-assembly of peptide amphiphiles: From molecules to nanostructures to biomaterials. Biopolymers 2010; 94(1): 1-18.

[8] Fung SY, Yang H, Chen P. Sequence effect of selfassembling peptides on the complexation and in vitro delivery of the hydrophobic anticancer drug ellipticine. PLoS One 2008; 3(4): e1956.

[9] Colombo G, Soto P, Gazit E. Peptide self-assembly at the nanoscale: A challenging target for computational and experimental biotechnology. Trends Biotechnol 2007; 25(5): 211-8.

[10] Webber MJ, Tongers J, Newcomb CJ, et al. Supramolecular nanostructures that mimic VEGF as a strategy for ischemic tissue repair. Proc Natl Acad Sci U S A 2011; 108(33): 13438-43.

[11] Soukasene S, Toft DJ, Moyer TJ, et al. Antitumor activity of peptide amphiphile nanofiber-encapsulated camptothecin. ACS Nano 2011; 5(11): 9113-21.

[12] Holmes TC, de Lacalle S, Su X, Liu G, Rich A, Zhang $S$. Extensive neurite outgrowth and active synapse formation on self-assembling peptide scaffolds. Proc Natl Acad Sci U S A 2000; 97(12): 6728-33.

[13] Gelain F, Bottai D, Vescovi A, Zhang S. Designer selfassembling peptide nanofiber scaffolds for adult mouse neural stem cell 3-dimensional cultures. PLoS One 2006; 1: e119.

[14] Beniash E, Hartgerink JD, Storrie H, Stendahl JC, Stupp SI. Self-assembling peptide amphiphile nanofiber matrices for cell entrapment. Acta Biomater 2005; 1(4): 387-97.

[15] Marrink SJ, Risselada HJ, Yefimov S, Tieleman DP, de Vries AH. The martini force field: Coarse grained model for biomolecular simulations. J Phys Chem B 2007; 111(27): 7812-24.

[16] Monticelli L, Kandasamy SK, Periole X, Larson RG, Tieleman DP, Marrink SJ. The martini coarse-grained force field: Extension to proteins. J Chem Theory Comput 2008; 4(5): 819-34.

[17] Langham AA, Waring AJ, Kaznessis Y. Comparison of interactions between beta-hairpin decapeptides and sds/dpc micelles from experimental and simulation data. BMC Biochem 2007; 8(1): 11.

[18] Stendahl JC, Rao MS, Guler MO, Stupp SI. Intermolecular forces in the self-assembly of peptide amphiphile nanofibers. Adv Funct Mater 2006; 16(4): 499508. 